P 093 - Comparison of three bipedal tasks to quantify contribution of proprioception to postural stability in healthy children and adolescents : Helsinki University Central Hospital, HUH Children and Adolescents

Ylitalo, E.

2018

Ylitalo , E , Mäenpää , H \& Piitulainen , H 2018 , ' P 093 - Comparison of three bipedal tasks to quantify contribution of proprioception to postural stability in healthy children and adolescents : Helsinki University Central Hospital, HUH Children and Adolescents ' , Gait \& Posture , vol. 65 , pp. 382-383 . https://doi.org/10.1016/j.gaitpost.2018.07.027

http://hdl.handle.net/10138/301364

https://doi.org/10.1016/j.gaitpost.2018.07.027

publishedVersion

Downloaded from Helda, University of Helsinki institutional repository.

This is an electronic reprint of the original article.

This reprint may differ from the original in pagination and typographic detail.

Please cite the original version. 
Short communication

\title{
P 093 - Comparison of three bipedal tasks to quantify contribution of proprioception to postural stability in healhty children and adolescents
}

\author{
E. Ylitalo ${ }^{\mathrm{a}, *}$, H. Mäenpääb ${ }^{\mathrm{b}}$ H. Piitulainen ${ }^{\mathrm{c}}$ \\ ${ }^{\text {a } U n i v e r s i t y ~ o f ~ H e l s i n k i ~ a n d ~ H e l s i n k i ~ U n i v e r s i t y ~ C e n t r a l ~ H o s p i t a l, ~ H U H ~ C h i l d r e n ~ a n d ~ A d o l e s c e n t s, ~ H e l s i n k i, ~ F i n l a n d ~}$ \\ ${ }^{\mathrm{b}}$ Helsinki University Central Hospital, HUH Children and adolescents, Helsinki, Finland \\ ${ }^{\mathrm{c}}$ Aalto University and Helsinki University Central Hospital, Department on Neuroscience and Biomedical Engineering, Helsinki, Finland
}

\section{A R T I C L E I N F O}

\section{Keywords:}

Adolescents

Balance

Bipedal

Children

Postural sway

Static

\begin{abstract}
A B S T R A C T
Static postural sway can be quantified as variation in body's center of force (COF) position across time using a plantar pressure plate. We aimed to compare capability of three clinically feasible bipedal tasks to extract the contribution of proprioception to postural stability. We measured the postural sway of 24 healthy volunteers (age range 10.2-17.6 years) with a plantar-pressure plate with three different standing tasks: (1) normal standing, (2) normal standing on soft foam, and (3) feet together standing. Each task was repeated eyes closed to emphasize the contribution of proprioception on maintaining the postural stability. The effect of closing the eyes varied among the tasks, and was greatest in the feet together standing task, possibly due to greater proprioceptive demands in the more difficult tasks. It appears that standing feet together is a potential task for quantifying contribution of proprioception to postural stability.
\end{abstract}

\section{Introduction}

Static postural sway can be quantified as variation in body's center of force (COF) position across time using force or plantar pressure plates. Control of posture relies on visual, vestibular and somatosensory afference. In the somatosensory modality, both tactile and proprioceptive ("movement sensors") afference are involved. Postural stability develops during childhood and adolescence, but is likely not matured at age of 14 years [1]. The role of proprioception for the postural control in developing system is largely unknown. This is a preliminary study to optimize a protocol for our upcoming more extensive study to examine postural stability during development.

\section{Research question}

We aimed to compare capability of three clinically feasible bipedal tasks to extract the contribution of proprioception to postural stability.

\section{Methods}

We measured the postural sway of 24 healthy volunteers (age range 10.2-17.6 years, mean 13.7 years) with a plantar-pressure plate (RSscan, Belgium) at 33 samples/s. Subjects stood eyes open as still as possible for $30 \mathrm{~s}$ (per task) during (1) normal standing, (2) normal standing on soft foam, and (3) feet together standing. Each task was repeated eyes closed to emphasize the contribution of proprioception on maintaining the postural stability. Mean distance of center of force (from sample to sample) was computed for both mediolateral and anteroposterior directions, and for their resultant. The absolute difference in the postural sway between eyes-closed and eyes-open conditions was also extracted (Fig. 1).

\section{Results}

The normal standing (eyes open and closed) was more stable compared to foam and feet together standing tasks ( $\mathrm{p}<0.001$ for both). In the eyes-open condition, postural sway was $48 \%$ and $54 \%$

\footnotetext{
* Corresponding author.

E-mail address: elina.ylitalo@hus.fi (E. Ylitalo).
} 

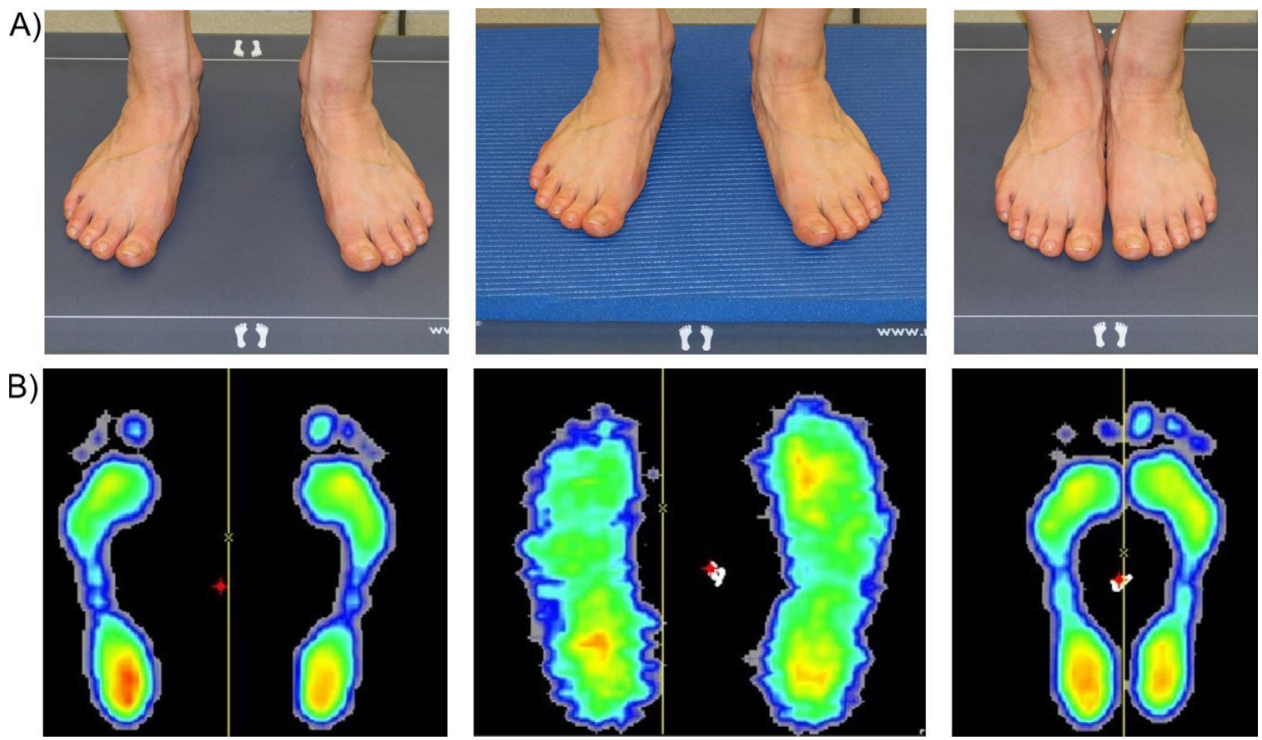

Fig. 1. A) Standing tasks: Normal standing on firm surface, normal standing on soft foam and standing feet together on firm surface. B) Pressure distribution from the above tasks.

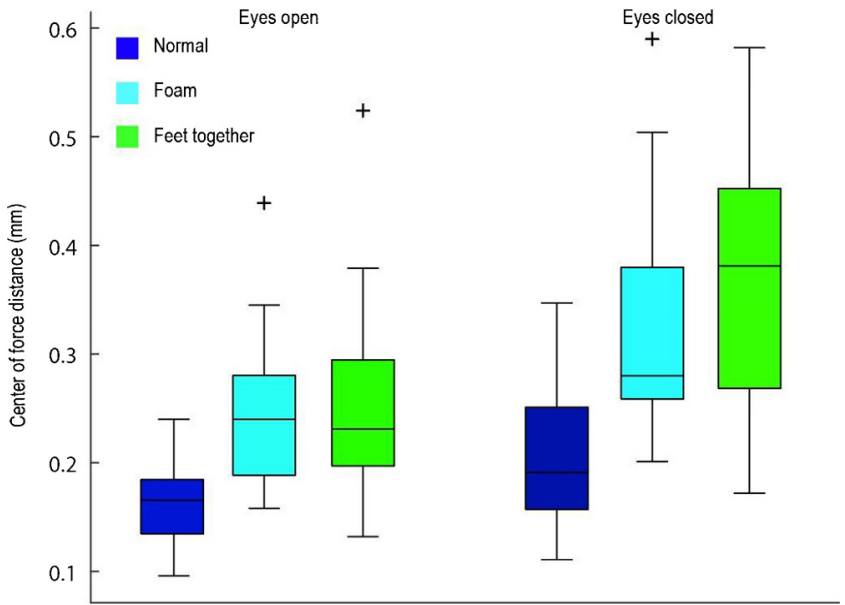

Fig. 2. Postural sway in the standing tasks eyes open and closed.

higher in foam and feet together standing respectively, and in eyesclosed condition, $58 \%$ and $81 \%$ higher than in normal standing. When the eyes were closed the postural sway increased by $25 \%, 33 \%$ and $47 \%$ in normal, foam and feet together standings respectively (t-values 4.98, 5.81 and 5.76 respectively, and $\mathrm{p}<0.001$ for all). The effect of closing eyes was $171 \%$ and $40.3 \%$ stronger during the feet together standing compared to normal $(\mathrm{p}=0.004)$ or foam $(\mathrm{p}=0.085)$ standing (Fig. 2).

\section{Discussion}

As expected, the postural sway was largest in the feet together standing, and was affected the most by closing the eyes. The effect of closing the eyes varied among the tasks, possibly due to greater proprioceptive demands in the more difficult tasks. Based on absolute amount of change in postural sway and statistical t-values, it appears that standing feet together is a potential task for quantifying contribution of proprioception to postural stability. This task is also feasible for clinical examination, e.g., to follow postural stability during treatment or after surgical operations etc.

\section{Disclosure statement}

This abstract is financially supported by grants from the Academy of Finland (grants \#296240 and \#304294) Jane and Aatos Erkko Foundation to Harri Piitulainen.

\section{References}

[1] M. Barrozzi, et al., Reliability of postural control measures in children and young adolescents, Eur. Arch. Otorhinolaryngol. 271 (2014) 2069-2077. 